\title{
Numerical Investigation and Analysis of the Unsteady Supercavity Flows with a Strong Gas Jet
}

\author{
X. Y Zhao, M. Xiang, H. C. Zhou and W. H. Zhang ${ }^{\dagger}$ \\ College of Aerospace Science and Engineering, National University of Defense Technology, Changsha \\ 410073, P.R. China
}

†Corresponding Author Email: xiangmin333@hotmail.com

(Received June 29, 2019; accepted December 31, 2019)

\begin{abstract}
Artificial supercavitation is one of the most prospective technique for underwater drag reduction, but it still faces some unsolved roadblocks, like cavity stability, noise, power etc. This article aims at investigating the instability of cavity with the strong jet impingement, and analyzing jet behavior in restricted space. A multiphase model using coupled VOF and level set method is adopted to capture the gas-liquid interface. By changing the position of jet nozzle exit and the jet intensity, a series of numerical simulation is performed. Firstly the transient evolution of the cavity interface under the effect of the high-speed jet is obtained. Numerical model is validated by comparing with the experimental data. Then the criterion to determine the transition between different jet/cavity interaction mechanisms is established based on a non-dimensional distance parameter. Furthermore, the entrainment mechanism inside the cavity is analyzed and provides useful insights on enhancing the cavity stability with strong jets.
\end{abstract}

Keywords: Jet, Supercavity; Instability; CLSVOF; Numerical research.

\section{NOMENCLATURE}

$\begin{array}{ll}A & \text { cross sectional area } \\ a & \text { turbulence coefficient } \\ C x & \text { drag coefficient of the cavitator } \\ C q & \text { gas ventilation coefficient } \\ D c & \text { maximum diameter of the supercavity } \\ D n & \text { cavitator diameter } \\ F r & \text { Froude number } \\ \mathrm{g} & \text { gravity } \\ \bar{J} & \text { non-dimensional number of the jet } \\ & \text { momentum flux } \\ L c & \text { length of the supercavity } \\ L_{1} & \text { potential core length of the jet } \\ L_{2} & \text { recirculation region length of the jet } \\ \dot{m}_{\text {jet }} & \text { mass flow rate of the jet } \\ \bar{P} & \text { non-dimensional number of the ratio of } \\ & \text { the free-stream total pressure to that of } \\ & \text { the jet } \\ p_{\infty} & \text { free liquid flow pressure } \\ p_{C} & \text { pressure inside the supercavity }\end{array}$

\section{INTRODUCTION}

Cavitation is a dynamic phenomenon that occurs when underwater vehicles travel with such a high speed that the pressure around the vehicle surface

$\begin{array}{ll}Q_{D} & \text { mass flow rate of the gas ventilation } \\ Q_{R} & \text { reflux mass flow rate } \\ \bar{S}_{0} & \text { non-dimensional distance parameter } \\ S_{D} & \text { cone cavitator bottom area } \\ S_{j e t} & \text { nozzle exit area } \\ U_{\infty} & \text { liquid flow velocity } \\ U_{C} & \text { centerline axial mean velocity } \\ U_{j e t} & \text { mean jet velocity at the nozzle exit } \\ W_{c} & \text { cavitator drag } \\ \pi \alpha & \text { half top angle of a cone } \\ \alpha_{g} & \text { volume fraction of the gas phase } \\ \varphi(x, t) & \text { level-set function } \\ \rho & \text { density } \\ \sigma_{c} & \text { cavitation number }\end{array}$

decrease to the vapor saturation pressure of the liquid. High drag reduction could be achieved when the vehicle is entirely covered by the cavity which is defined as supercavitation. However,5 it is difficult to achieve supercavitation under natural conditions. 
Reichardt (1946) firstly used the method of artificial air injection to form a steady cavity that covered the entire surface of an object. That pioneering work provided the possibility for engineering application of drag reduction by cavitation. Afterwards an amount of works that contain theoretical and experimental work on ventilated cavitation were performed. The empirical formulas of the stable cavity shape and the ventilation rate demand for supercavity formation without external perturbations have been built. In one word, ventilated cavity flows without jet mainly depends on several nondimensional fundamental parameters: such as cavitation number, $\sigma_{c}$, Froude number, $F r$, and the gas ventilation coefficient, $C q$.

$$
\begin{aligned}
& \sigma_{c}=\frac{2\left(p_{\infty}-p_{c}\right)}{\rho_{\infty} U_{\infty}^{2}} \\
& F r=\frac{U_{\infty}}{\sqrt{g D_{n}}} \\
& C_{\mathrm{q}}=\frac{Q_{D}}{\rho_{\infty} U_{\infty} D_{n}^{2}}
\end{aligned}
$$

In those relation, $p_{\infty}, \rho_{\infty}$ and $U_{\infty}$ denote the free liquid flow pressure, the liquid density and velocity respectively; $\quad p_{c}$ is the pressure inside the supercavity; $D n$ is the cavitator diameter; and $Q_{D}$ refers to the gas ventilation mass flow rate.

In order to achieve high drag reduction efficiency, it is always required a stable cavity shape. However the cavitation stability could be disturbed by many factors. As the jet engine is considered as the most prospect propulsion system for the supercavitating vehicle, recently, the effects of the gas jet from exhaust nozzle on an established cavity has got more and more attention. Paryshev (2006) gave an approximate mathematical models of cavity when it closes on a central liquid or incompressible gas jet. According to his theory, the effect of jet on the cavity was governed by two non-dimensional numbers: $\bar{P}$, the ratio of the stagnation pressure of the surrounding flow to that of the jet, and $\bar{J}$, the ratio of the jet momentum flux to the cavitator drag, which are defined as follows:

$$
\begin{aligned}
& \bar{P}=\frac{\rho_{\infty} U_{\infty}^{2}}{\rho_{j e t} U_{j e t}^{2}} \\
& \bar{J}=\frac{\dot{m}_{j e t} U_{j e t}}{W_{c}}
\end{aligned}
$$

Where $U_{\text {jet }}$ denotes the mean jet velocity at the nozzle exit; $\dot{m}_{\text {jet }}$ denotes the mass flow rate of the jet; $W_{c}$ is the cavitator drag. Jet/supercavity interaction is divided into three categories: (1) when $\bar{P}>1$ and $\bar{J}<1 / 2$, the jet is totally obstructed by the cavity, and returns into it. (2) when $\bar{P}>1$ and $\bar{J}>1 / 2$, the jet is divided into two parts, a portion goes out of the cavity while the other portion returns and inflates the cavity. (3) when $\bar{P}<1$, the jet isn't completely obstructed and leaves the cavity. Money (2015) conducted a series of experiments on jet-supercavity interaction in the $1.22 \mathrm{~m}$ dia. Garfield Thomas Water Tunnel. By changing the jet mass flow rates from 0 to $0.11 \mathrm{~kg} / \mathrm{s}$, experiments were performed at two tunnel speeds and for two nozzle diameters. Collected data well supported Paryshev's theory models. Kinzel et al. (2017) used a high-fidelity CFD method to evaluate the modeling developed by Paryshev. CFD results were in consistent with the experimental data and showed that Paryshev's Model should be generalized to account for stagnation pressure losses of the jet. $\mathrm{Xu}$ Hao et al. (2018) carried out an experimental study on the supersonic gaseous jet induced tail cavity at the wake of a revolution body, and investigated the transient evolution characteristics and forming mechanism. According to the observation, the induced cavities had four forms: foamy, intact, partially break, and pulsating foamy closure type. About the recent research progress on the unsteady supercavity, Zou et al. (2010) gave a gas-leakage rate formula for the unsteady ventilated supercavity and verified its validity. Based on Logvinovich model, Zou et al. (2013) studied the relationship between the shedding frequency and the velocity disturbance for the unsteady supercavity flows. Karn et al. (2016) conducted an important study on closure mechanisms for the unsteady ventilated supercavity. These unstable closure modes were either observed at the transition of two stable closure modes or at superfluous ventilation rates. Sun et al. (2019) carried out a numerical investigation on the shedding mechanism for the ventilated partial cavitating flows, and analyzed how the vortex structures were created and developed in the cavity shedding process.

Considering that enough propulsion is always required to insure that underwater vehicles could run steadily in a high speed, we prefer to concern the supercavtiy flows with a strong gas jet that could provide thrust no less than the cavitator $\operatorname{drag}(\bar{J}>1$, or $\bar{J} \approx 1)$. With sufficient momentum, such cavities could experience instability, pulsation and collapse under certain circumstances. This article aims at investigating the instability of cavity with the strong jet impingement, and analyzing jet behavior in restricted space based on theoretical and numerical methods. Besides jet strength, the distance between the nozzle exit and cavity closure is considered as a very important parameter in jet/supercavity interaction. As the relative position varies, it may lead to diverse cavity surface evolution and different jet entrainment effect in cavity. In this paper, simulation for a supercavity case without jet will be firstly carried out under different ventilation flow rate and comparison with the experimental data will be carried out to validate the numerical model. Then, by changing jet nozzle exit, a series of numerical simulation will be performed. The effect of the relative position on the jet/cavity interaction will be analysed to provide useful methods in enhancing the stability of the ventilated cavity. 


\section{Theoretical Analysis OF The JET/CAVITY INTERACTION}

\subsection{Analysis of the Key Parameters}

Parametric analysis is a necessary task before numerical simulation. Figure 1 shows all important parameters that may have an impact on the jet/cavity interaction including the jet parameter, the cavitation parameter and the geometry parameters. Figure 2 shows the criterion for the jet/cavity closure interaction mechanism by Paryshev and Money and the improved criterion for the cavity gas-leakage pattern under jet effects. Obviously, the parameter $\bar{J}$ and $\bar{P}$ represent implicitly the jet strength in the above criterions, but other parameters, like nozzle geometry and relative position, are not reflected in the discrimination mechanism. Therefore we try to expand Paryshev's theory and enrich the dimensionless parameters to describe the jet/cavity interaction, in order to build criterions for the different interface evolution mechanism change.

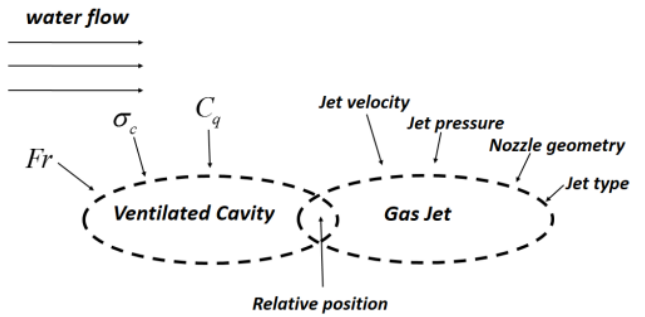

Fig. 1. Key parameters in the jet/cavity interaction.

First of all, the correlation between two nondimensional numbers $\bar{P}, \bar{J}$ and the ratio of the nozzle exit area to the cavitator bottom area is derived.

According to drag and mass flow rate formula equations, we could primitively get:

$W_{c}=1 / 2 \rho_{\infty} U_{\infty}^{2} S_{D} C_{x}$

$\dot{m}_{\text {jet }}=\rho_{\text {jet }} U_{j e t} S_{j e t}$

In those relations, $S_{D}$ and $S_{j e t}$ denote the cone cavitator bottom area and the nozzle exit area respectively. In combination with Eqs.(4) and (5), furthermore, we could get:

$\bar{J} \cdot \bar{P}=\frac{2}{C_{x}} \frac{S_{j e t}}{S_{D}}$

The above relation shows that the effect of dimensionless nozzle exit area is reflected implicitly by the important parameter $\bar{J}$ and $\bar{P}$. Despite all this, it could provide some meaningful advice for supercavity vehicle design. When thrust $(\bar{J})$ is constant, we should improve $S_{j e t} / S_{D}$ to insure $\bar{P}>1$, so that the cavity is steady based on
Paryshev's theory. Figure 3 shows the improved criterion for the cavity gas-leakage pattern under jet effects. Figures 4 and 5 shows the gas-leakage pattern in different mechanisms, when $\bar{P}>1$, gas leaks out of the cavity in the way of the axial flow pattern; when $\bar{P}<1$, gas leaks out of the cavity in the way of the periodic pulsation pattern.

\subsection{Review of Jet Dynamic}

In order to understand the internal flow field structure of the cavity with a strong jet, we firstly review of gas jet dynamic knowledge. In general, the jets are classified into two types: free jet and confined jet (as shown in the figure 6 and 7) on the basis of the surrounding environment's effects. The free jet, often exists in an unlimited space and acts as a turbulent free jet. In this paper, we use a circle exit of the converging nozzle. Previous experimental research by Albertson et al. (1950) and Schwarz (1963) on the gas jet shows that, when the jet leaves from the orifice and enters into a quiescent environment, the velocity gradient results in the generation of turbulence eddies, and the jet would entrains the surrounding gas and moves forward together. Meanwhile, jet boundary spreads freely in the radial direction. The whole jet development process is composed of two parts: the zone of flow establishment and the zone of established flow. Near the nozzle exit, a triangular area which is not affected by the entrainment and still maintain the original exit velocity is defined as the potential core. The regions between the potential core and the jet boundary is often called the shear layer (or mixing layer). The potential core length $\left(L_{1}\right)$ depends on the geometry shape of convergent nozzle exit.

$L_{1}=0.671 \frac{D_{j e t}}{2 a}$

Where $D_{j e t}$ is the diameter of the nozzle exit, and $a$ is the turbulence coefficient which could be obtained through experiment, it is often given as a range value of $0.053 \sim 0.071$ for circle nozzle.

For a confined jet, Kandakure et al. (2008) and Ball et al. (2012) studied on the jet behavior. A recirculation flow pattern is established because the amount of surrounding gas is not enough for the entrainment in presence of tank wall. Therefore, the jet radius firstly increases and then decreases. The jet boundary is featured and has experienced three stages: free expansion (section I to section II), restricted expansion (section II to section III), and contraction (section III to section IV). The presence of the walls will lead to the higher shear rates which increases the turbulence than that of free jet. Behind the recirculation region, the flow filed turns into the turbulent vortex region. The whole recirculation region length (section I to section IV) $L_{2}$ is:

$L_{2}=3.58 \sqrt{A}+\frac{D_{j e t}}{a}\left(0.147 \frac{\sqrt{A}}{D_{j e t}}-0.133\right)$ 


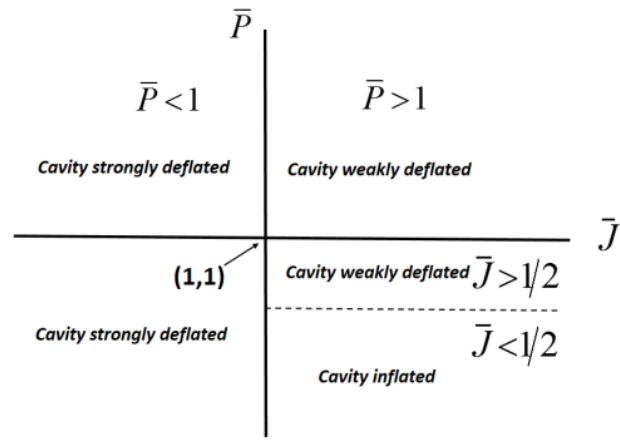

Fig. 2. Criterion for the jet/cavity closure interaction mechanism by Paryshev and Money.

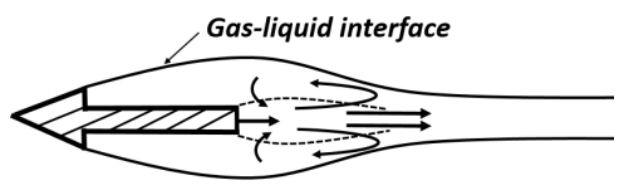

Fig. 4. Structure of the cavity closure onto a central jet of $\bar{P}>1$

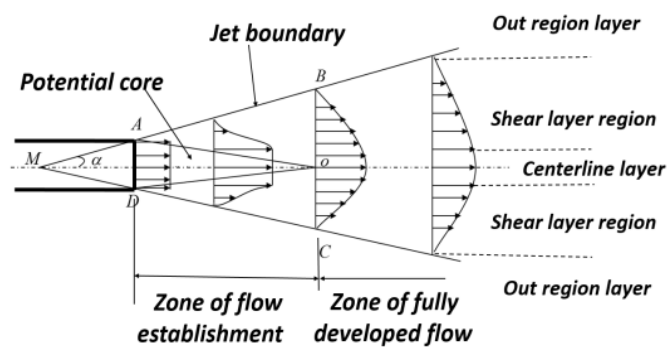

Fig. 6. Schematic of the turbulent round free jet.

Where $A$ denotes the cross sectional area of the external tank wall.

The above jet dynamic knowledge could help us to understand jet/surpercavity interaction. The jet structure inside the cavity is similar to the confined jet, the only difference is that cavity interface is not fixed walls and relates to complex interface evolution. In one word, gas leakage ways induced by jet entrainment and cavity interface instabilities jointly determine the interface evolution mechanism. Under certain circumstances the jet entrainment would result in the atrophy of the cavity when the cavity doesn't have enough gas to maintain the cavity shape. On the one hand, the intensity of the entrainment effect is related to the jet strength. For an established supercavity, when jet momentum $\bar{J}$ is small, and the velocity gradient is low, the jet couldn't bring gas away from the cavity. As $\bar{J}$ increases to a certain value, due to the enhancement in velocity gradient shear, the jet starts to entrain surrounding gas and leave from the

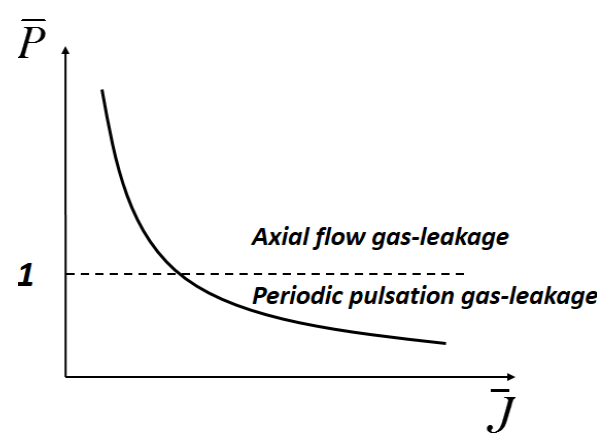

Fig.3. Improved criterion for the cavity gasleakage pattern under jet effects.

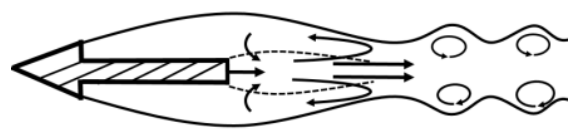

Fig. 5. Structure of the cavity closure onto a central jet of $\bar{P}<1$.

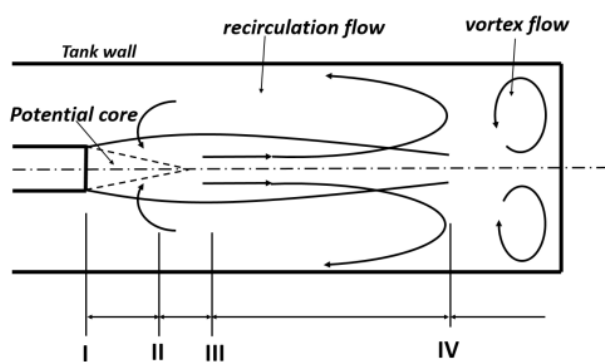

Fig. 7. Schematic of the turbulent round confined jet.

cavity together. The more gas that maintains the cavity are brought away, the more the cavity shrinks. As $\bar{J}$ continues to increase, the strengthened entrainment effect may result in a pinch-off, pulsating, and unsteady supercavity.

On the other hand, the strength of the entrainment effect is also related to the relative position. When the nozzle exit is nearer to the cavity closure, the shear layer (or mixing layer) zone of jet would diminish. It means that the surrounding gas entrained by jet decrease and may mitigate the negative effects caused by the jet.

\subsection{Several Situations of Jet/Cavity Interaction}

For an established supercavity and a constant- $\bar{J}$ jet, the distance between the jet nozzle exit and the point of cavity closure $\left(S_{0}\right)$ is speculated to be an important parameter that impacts surface evolution mechanism for the cavity. However, in view of the 

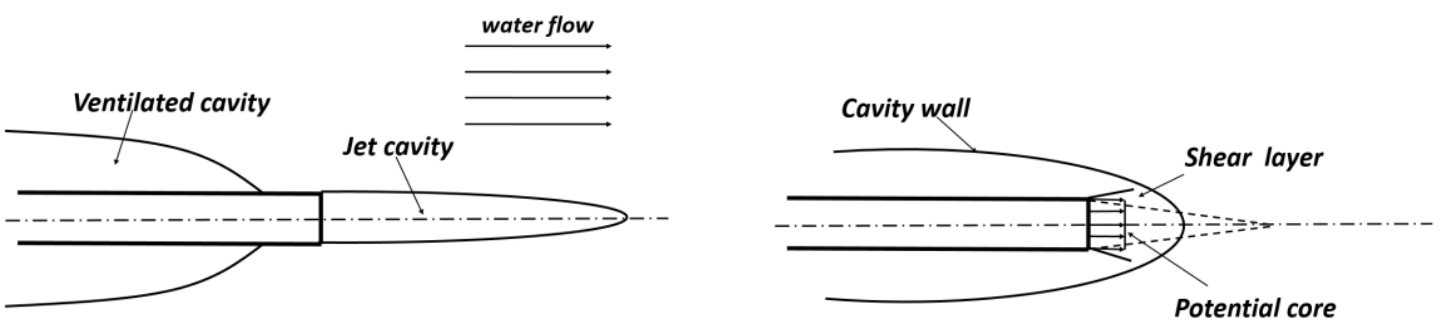

Situation1

Situation 2

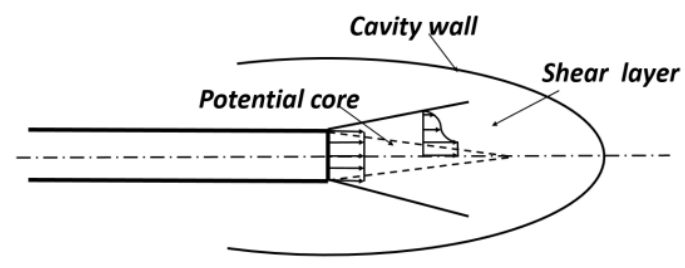

Situation 3

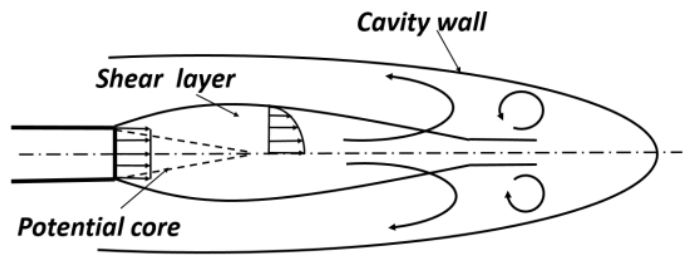

Situation 4

Fig.8. Criterion for the jet/cavity interaction mechanism of varying relative position.

asymmetric characteristics of the cavity surface at low $\mathrm{Fr}$ number, the jet would firstly interact with the lower cavity surface, rather than the closure region due to the buoyance effect, so we redefine the distance parameter which represents the relative position of jet/cavity, that is the axis length of the nozzle outlet to the lower cavity surface. We also give the normalized form as:

$$
\overline{S_{0}}=S_{0} / D_{n}
$$

When the relative position varies, several situations of jet/cavity interaction are analyzed as follows, Situation 1: the ventilated cavity closes on the slender body and has little connection with the tail cavity induced by jet.

Situation 2: when $0<S_{0}<L_{1}$ ( or $0<\overline{S_{0}}<L_{1} / D_{n}$ ), the nozzle exit is nearer to the cavity closure and the cavity tail closes in the jet's potential core, the jet entrainment effect in cavity is very weak and almost negligible.

Situation 3: when $L_{1}<S_{0}<L_{2} \quad$ (or $L_{1} / D_{n}<\overline{S_{0}}$ $\left.<L_{2} / D_{n}\right)$, the cavity tail closes at the location outside the potential core, the jet entrainment effect is enhanced.

Situation 4: when $S_{0}>L_{2}\left(\right.$ or $\left.\overline{S_{0}}>L_{2} / D_{n}\right)$, the entire shear area of the jet is covered by the cavity, and the jet would entrain intensively the surrounding gas from the cavity. We focus on Situation 2 to Situation 4 in our research. The strong entrainment and dissipation of turbulent vortexes bring about the internal pressure and velocity pulsation inside the cavity, eventually resulting that the cavity fail to maintain a stable state, thus requiring the formation of a new interface state.

\section{NUMERICAL MethodS}

\subsection{Computational Methodology}

In this manuscript, we use a homogeneous multiphase model based on FLUENT platform. A single set of momentum equations is shared by the fluids, and all material properties of the fields would be determined by the component phases, so long as the volume fraction of each phase is calculated at each control cell. There are two phases in the fluid field, an incompressible liquid phase for the external field and an incompressible noncondensable gas phase for the ventilation and jet. The liquid-gas interface is captured by a method which coupled VOF with level set. It is reasonable cognition that surface tension and turbulence model is important, the classical standard k-epsilon model and surface tension model are used in our simulation.

\subsubsection{Governing Equation}

The governing continuity equation and momentum equation of a homogeneous multiphase flow are given as follows:

$$
\begin{aligned}
& \frac{\partial \rho}{\partial t}+\frac{\partial}{\partial x_{i}}\left(\rho u_{i}\right)=0 \\
& \frac{\partial\left(\rho_{m} \boldsymbol{u}\right)}{\partial t}+\nabla \bullet\left(\rho_{m} \boldsymbol{u} \boldsymbol{u}\right)=-\nabla p+\nabla \bullet\left[\mu_{m}\left(\nabla \boldsymbol{u}+\nabla \boldsymbol{u}^{\mathrm{T}}\right)\right]+\rho_{m} \boldsymbol{g}+\boldsymbol{F}_{s}
\end{aligned}
$$

The density and viscosity coefficient of the mixture phase respectively is:

$$
\begin{aligned}
& \rho_{m}=\rho_{l}\left(1-\alpha_{g}\right)+\rho_{g} \alpha_{g} \\
& \mu_{m}=\mu_{l}\left(1-\alpha_{g}\right)+\mu_{g} \alpha_{g}
\end{aligned}
$$


Where $\alpha_{g}$ represents the volume fraction of gas phase, on the other hand $1-\alpha_{g}$ could represents the volume fraction of liquid phase.

\subsubsection{Coupled VOF and Level Set Method:}

In the method of coupled VOF and level-set (CLSVOF) proposed by the Bourlioux (1995) and Sussman and Puckett (2000), the interface is captured and tracked by solving the volume fraction equation and level-set function together. The main idea is to benefit from the advantage of each strategy, which is to ensure mass conservation with the VOF method and to keep a fine description of the geometrical properties of the interface by the level set method. And the volume fraction equation for the gas phase is given as follows:

$\frac{\partial \alpha_{g}}{\partial t}+\nabla \cdot\left(\boldsymbol{u} \alpha_{g}\right)=0$

The cell domain of $0<\alpha_{g}<1$ contains the interface between the gas phase and the liquid phase.

The level-set function $\varphi(x, t)$ is defined as a signed distance between any point in the field and the interface. Accordingly, the interface is the zero level-set, $\varphi(x, t)$ and can be expressed as $\Gamma=\{x \mid \varphi(x, t)=0\}$ in a two-phase flow system:

$\varphi(x, t)= \begin{cases}+|d|, & x \in \text { the primary phase } \\ 0, & x \in \Gamma \\ -|d|, & x \in \text { the second phase }\end{cases}$

Where $d$ is the distance from the interface. In a given velocity field $\boldsymbol{u}$, solving a convection equation determines the evolution of the interface:

$$
\frac{\partial \varphi}{\partial x}+\nabla \cdot(\mathbf{u} \varphi)=0
$$

By nature of the transport equation of the level-set function Eq. (18), it is unlikely that the distance constraint $|\nabla \varphi|=1$ of is maintained after its solution. A re-initialization process is therefore needed for each time step. The geometrical interface construction method which is reliable on the values of the VOF and the level-set function is used here to reconstruct the interface. Namely, the VOF method provides the size of the cut in the cell where the likely interface passes through, and the gradient of the level-set function determines the direction of the interface.

\subsubsection{Surface Tension Model}

In momentum Eq.(13), $\boldsymbol{F}_{s}$ is the force arising from surface tension effects given by:

$\boldsymbol{F}_{s}=\sigma \kappa \delta(\varphi) \boldsymbol{n}$

Where $\sigma$ is the surface tension coefficient and equals to $0.072 \mathrm{~N} / \mathrm{m}$ at normal temperature. And $\delta(\varphi)$ function is defined as:

$\delta(\varphi)= \begin{cases}0, & |\varphi| \geq \varepsilon \\ \frac{1+\cos (\pi \varphi / \varepsilon)}{2 \varepsilon}, & |\varphi|<\varepsilon\end{cases}$

Where $\varepsilon$ is the thickness of the gas-liquid interface. The normal, $\boldsymbol{n}$, and curvature, $\boldsymbol{\kappa}$, of the interface can be given as:

$$
\kappa=\left.\nabla \cdot \frac{\nabla \varphi}{|\nabla \varphi|}\right|_{\varphi=0}, \quad \boldsymbol{n}=\left.\frac{\nabla \varphi}{|\nabla \varphi|}\right|_{\varphi=0}
$$

\subsection{Physical Model, Mesh and Boundary Condition Description}

The object of simulation which used to research on jet/cavity interaction is derived from Money's experimental model (2015). The model consists of a 30 -degree, $31.75 \mathrm{~mm}$ diameter cone cavitator at the head of the slender body and a converging circular nozzle in the rear. The model has the geometrical characteristic of large cavitator, short body and small nozzle exit, the ratio of nozzle exit area to cavitator bottom area, $S_{j e t} / S_{D}$, is approximately equal to 0.12 . We know that it is easy to form unsteady supercavity flows with a strong gas jet from the previous analysis. In order to investigate the effect of relative position, we have designed four different length size for the simulation model.

The computational mesh is showed in Fig.10. Grid refinement is carried out around the physical model body and in the nozzle tail domain. The number of mesh nodes is 1.7 million after mesh adaption analysis, which can better capture the interface variation of the cavity and the development of the jet morphology.

The boundary conditions are applied as follows: the velocity inlet and pressure outlet boundary conditions are used for the distribution of the incoming flow and outlet boundary, and the symmetric boundary condition is used for the symmetric surface. The non-slip wall boundary condition is used for the physical mode wall and the slip wall boundary condition is use for the far field. The ventilation and jet ports use mass flow inlet boundary.

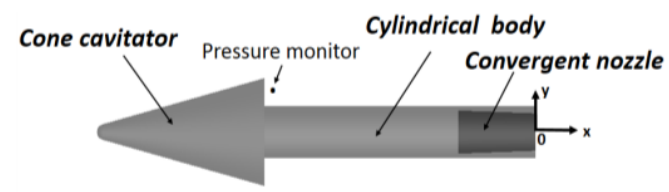

Fig. 9. Physical model of simulation.

\subsection{Validation for a Supercavitating Case}

Firstly we have done a validation work of steady supercavity without jet by comparing the CFD results with theory data. Guzevsky (1973) gave empirical formulas for the supercavity length, 
maximum diameter and drag coefficient of cone cavitator.

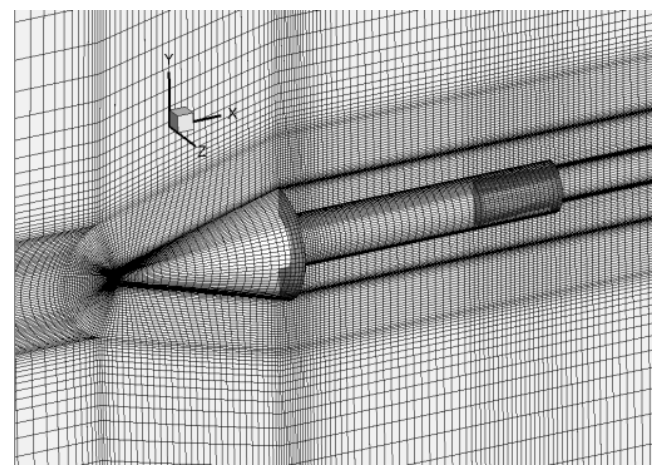

Fig. 10. The computational mesh.

$D_{c}=D_{n} \sqrt{\frac{C_{x}}{k \sigma_{c}}}$

$L_{c}=D_{n}\left[\frac{1.1}{\sigma_{c}}-\frac{4(1-2 \alpha)}{1+144 \alpha^{2}}\right] \sqrt{C_{x} \ln \frac{1}{\sigma_{c}}}$

$k=\frac{1+50 \sigma_{c}}{1+56.2 \sigma_{c}}$

$C_{x}=C_{x 0}+(0.524+0.672 \alpha) \sigma_{c} \quad 1 / 12 \leq \alpha \leq 1 / 2$

$C_{x 0}=0.5+1.81(\alpha-0.25)-2(\alpha-0.25)^{2} \quad 1 / 12 \leq \alpha \leq 1 / 2$

Where $D c$ and $D n$ are the theoretical maximum diameter of the supercavity cross section and the diameter of the cone cavitator; $C x$ represents the drag coefficient of the cone cavitator, $C x_{0}$ is the drag coefficient when cavitation number equals zero. $\pi \alpha$ is the half top angle of a cone, so $\alpha=1 / 12$ in this paper.

As shown in Fig.11 and table 1, under different ventilation flow rate, the average relative errors of the comparisons in the cavity maximum diameter, length and the drag coefficient are $2.25 \%, 8.7 \%$ and $5.1 \%$, respectively.

\section{NUMERICAL RESUltS DISCUSSIONS}

AND

In order to study the effect of $\bar{S}_{0}$ on jet/supercavity interaction, one way is to change the ventilation rate or the free stream velocity so that the cavity closure region closes to the nozzle exit, the other way is to elongate the model's body to make that the nozzle exit closes to the cavity closure region. We choose the latter because that could avoid introducing other variables when different $\bar{S}_{0}$ is analyzed. We firstly simulate a steady cavity in the condition of $F r=11.4, C q=0.16$. And then a strong jet with strength of $\bar{J}=1, \bar{P}=1.355$ and $\bar{J}=1.88, \bar{P}=0.72$ is generated. Thereafter, by changing the relative position of nozzle exit to the cavity with four different length of physical models, a series of numerical simulation of supercavitating flows is performed as shown in.

According to formula Eq. (9), the dimensionless jet potential core length is obtained:

$L_{1} / D_{n}=\frac{0.671}{2 a} \frac{D_{j e t}}{D_{n}}$

According to formula Eqs. (10) and (22), where A, the cross sectional area in the confined space is decided by the maximum cross sectional area of the cavity, the dimensionless recirculation region length of the jet is obtained:

$L_{2} / D_{n}=\left(3.58+\frac{0.147}{a}\right) \sqrt{\frac{\pi}{8}} \frac{D_{C}}{D_{n}}-\frac{0.133}{a} \frac{D_{\text {jet }}}{D_{n}}$

The theoretical value of $L_{1} / D_{n}$ and $L_{2} / D_{n}$ are 2.19 and 6.4 respectively. Figure 12 shows four groups of the relative position between nozzle exit and cavity. Combined with the discriminant criterion for the jet/cavity interaction mechanism with varying relative position (Fig. 8), these calculation cases could be reasonably classified: the case $1 \mathrm{a}$, case $1 \mathrm{~b}$, case $2 \mathrm{a}$ and case $2 \mathrm{~b}$ belong to situation 4 , the case $3 \mathrm{a}$ and case $3 \mathrm{~b}$ belong to situation3, and the case $4 \mathrm{a}$ and case $4 \mathrm{~b}$ belong to situation 2 .

\subsection{The cavity Interface Evolution Process}

Figures 13 and 14 show respectively the cavity interface transient evolution process for case $1 \mathrm{~b}$

$\left(\bar{P}<1 \quad\right.$ and $\left.\overline{S_{0}}>L_{2} / D_{n}\right) \quad$ and case $4 \mathrm{~b} \quad(\bar{P}<1$ and $\left.\overline{S_{0}}<L_{2} / D_{n}\right)$. For case $1 \mathrm{~b}$, a comparison is shown between CFD predictions and Money's experiment results (2015). The numerical methods are proved to be accurate in capturing interface motion for the cavity. It can be clearly seen from the CFD results that the cavity bubble pinch off and shed due to elongated morphology, while in the experiment, because of the strong instability of gasliquid interface and the mixing of water and gas, a large number of continuous broken bubbles are actually observed.

The cavity interface evolution process could be subdivided into two stages according to present simulation and experiment dates. During the first stage, the cavity experiences an expansion, necking, and shedding process with jet effects. The total cavity gets a reduction in volume by retraction, which is showed as the elastic behavior of cavity interface. Then, for the second stage, the cavity presents periodic gas-leakage in the tail and maintain this unsteady state of affairs continued. But for case $4 b$, the first stage is not obvious, after a period of temporary the cavity begins directly to leak gas in the way of the periodic pulsation pattern. The difference between two situations is that the former exists a fluid thread breakup phenomenon drove by the Plateau-Rayleigh instability explained by Eggers (1997), which is characterized by the elongation of the fluid shape forming thin, threadlike regions between larger nodules of fluid. 

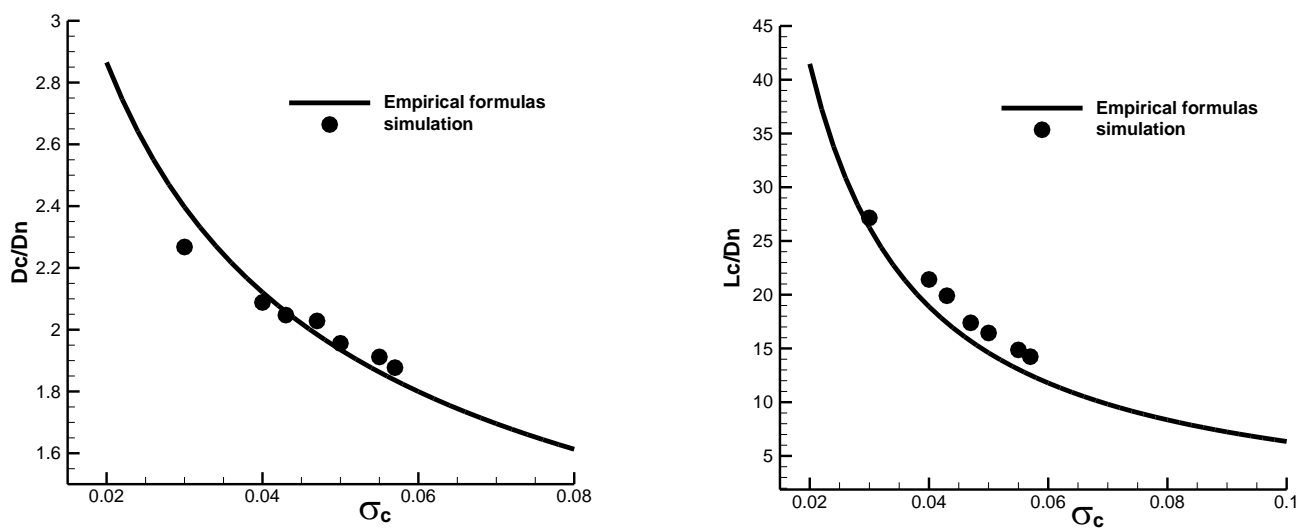

Fig. 1. Dimensionless maximum diameter and length of the cavity at different cavitation number.

Table 1 Drag coefficient of 30-degree cone cavitator

\begin{tabular}{|c|c|c|c|}
\hline $\begin{array}{c}\text { Ventilation } \\
\text { coefficient }\end{array}$ & $\begin{array}{c}\text { Cavitation } \\
\text { number }\end{array}$ & $\begin{array}{c}\text { CFD results } \\
C x\end{array}$ & Theory results $C x$ \\
\hline 0.12 & 0.057 & 0.177 & 0.176 \\
\hline 0.16 & 0.055 & 0.177 & 0.175 \\
\hline 0.32 & 0.050 & 0.175 & 0.172 \\
\hline 0.64 & 0.047 & 0.178 & 0.170 \\
\hline 0.96 & 0.043 & 0.179 & 0.168 \\
\hline 1.28 & 0.040 & 0.180 & 0.167 \\
\hline 2.56 & 0.030 & 0.188 & 0.160 \\
\hline
\end{tabular}

Table 2 Calculation cases of jet/cavity interaction with different relative position

\begin{tabular}{|c|c|c|c|c|c|}
\hline case & $U_{\infty}(\mathrm{m} / \mathrm{s})$ & $Q_{D}(\mathrm{~kg} / \mathrm{s})$ & $\dot{m}_{\text {jet }}(\mathrm{kg} / \mathrm{s})$ & $\bar{J}$ & $\bar{S}_{0}$ \\
\hline Case 1a & 6.36 & 0.00062328 & 0.00908307 & 1 & 10.90 \\
\hline Case 1b & 6.36 & 0.00062328 & 0.0124656 & 1.88 & 10.90 \\
\hline Case 2a & 6.36 & 0.00062328 & 0.00908307 & 1 & 7.56 \\
\hline Case 2b & 6.36 & 0.00062328 & 0.0124656 & 1.88 & 7.56 \\
\hline Case 3a & 6.36 & 0.00062328 & 0.00908307 & 1 & 2.83 \\
\hline Case 3b & 6.36 & 0.00062328 & 0.0124656 & 1.88 & 2.83 \\
\hline Case 4a & 6.36 & 0.00062328 & 0.00908307 & 1 & 1.26 \\
\hline Case 4b & 6.36 & 0.00062328 & 0.0124656 & 1.88 & 1.26 \\
\hline
\end{tabular}
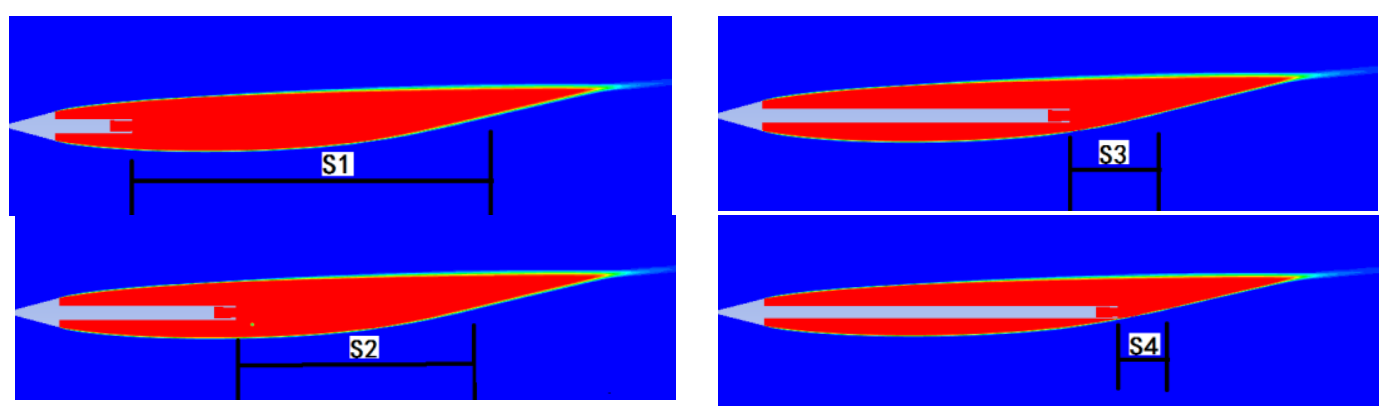

Fig. 2. Relative position between nozzle exit and cavity.

breakup of the fluid into small packets to minimize the system surface energy by reducing the surface area. Especially, the changes of the cavity length needs to be concerned. Further research is needed to make certain when the cavity would experience pinch-off. The distance parameter $\overline{S_{0}}=L_{2} / D_{n}$ may be the critical point.

In the cavity interface evolution process, two special moments are emphatically analyzed. The moment of $\mathrm{t}=0.15 \mathrm{~s}$ is that the cavity forms the final shape in the periodic varying state of growth and shedding. And the moment of $t=0.005 \mathrm{~s}$ is that the 


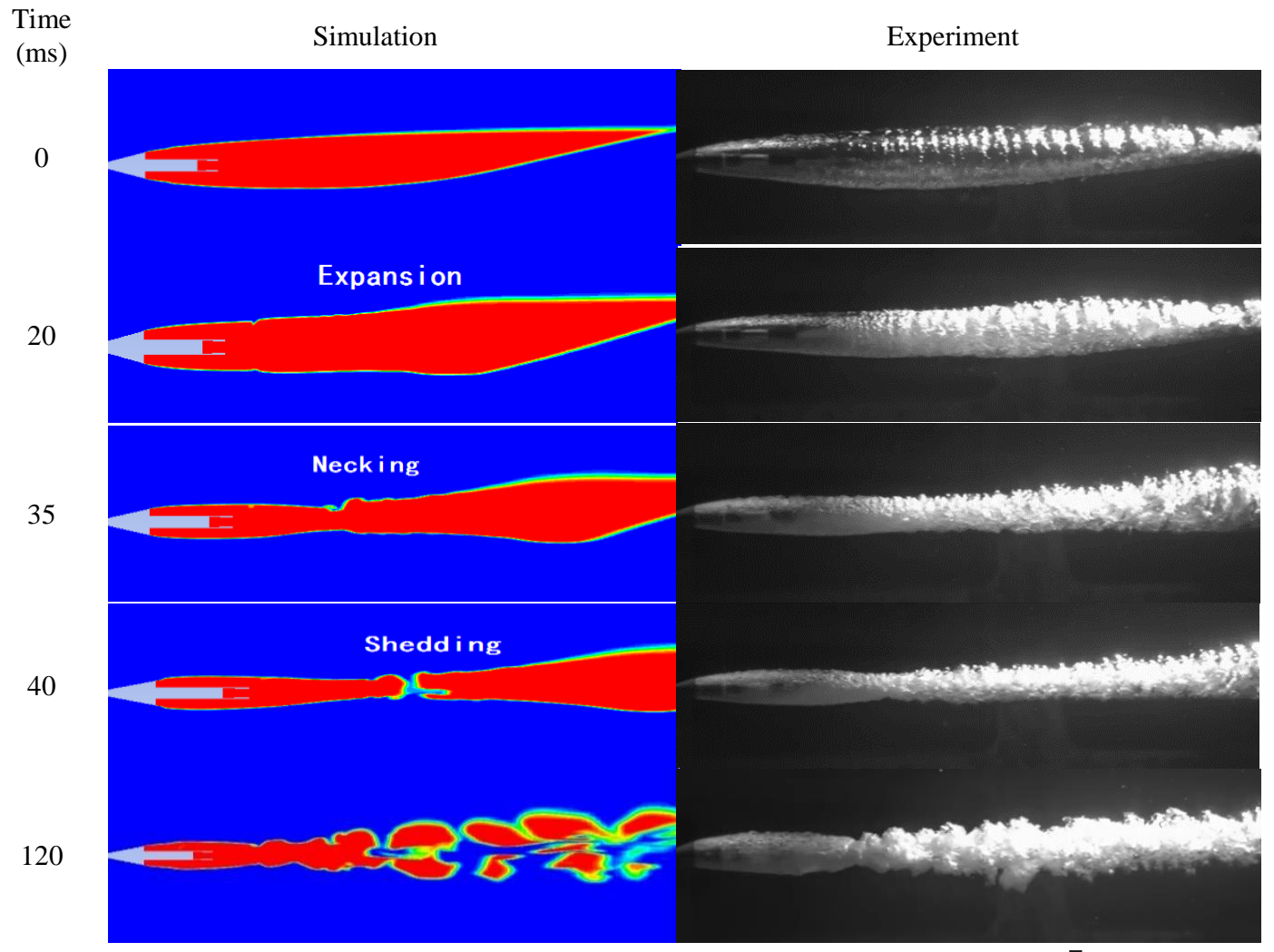

Fig. 3. Cavity interface evolution showed by the contours of volume fraction when $\bar{P}<1$ and $\overline{S_{0}}>L_{2} / D_{n}$ (blue regions: water, red regions: gas).

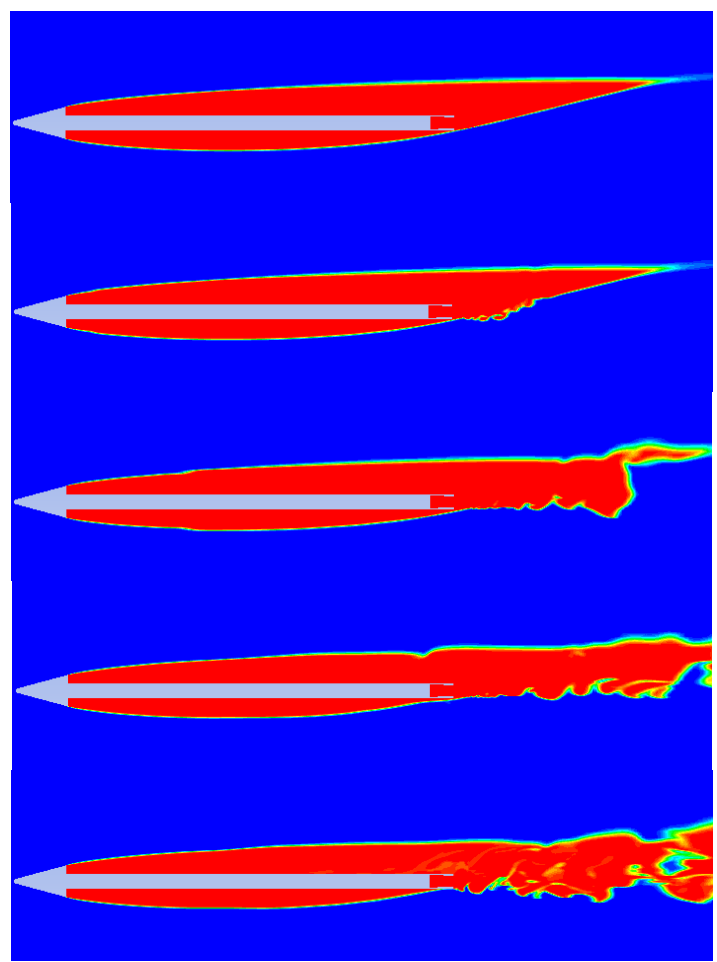

Fig. 4. Cavity interface evolution when $\bar{P}<1$ and $\overline{S_{0}}<L_{2} / D_{n}$.

jet has just formed but the cavity shape has not changed yet. Clearly describing the structural characteristics of the confined jet, the strength of the entrainment effect and it's influence on the quality change inside the cavity at this moment, that is meaningful to study the evolution mechanism of the cavity interface. 


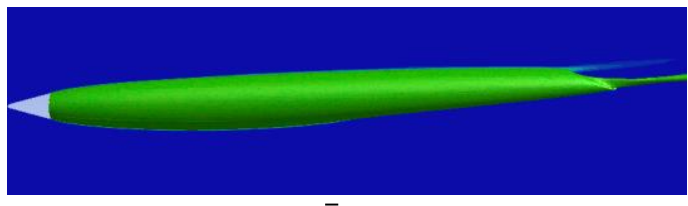

$\bar{J}=0$

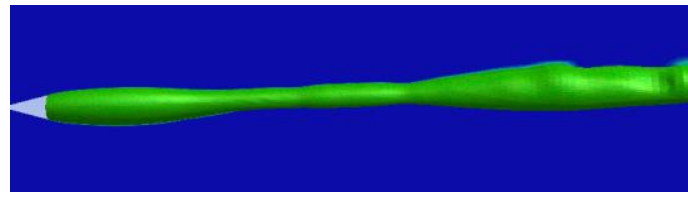

(1a) $\mathrm{S} 1_{-} \bar{J}=1$

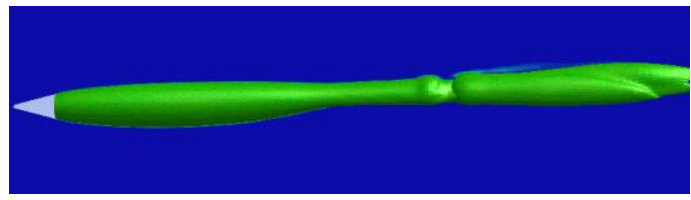

(2a) $\mathrm{S} 2 \_\bar{J}=1$

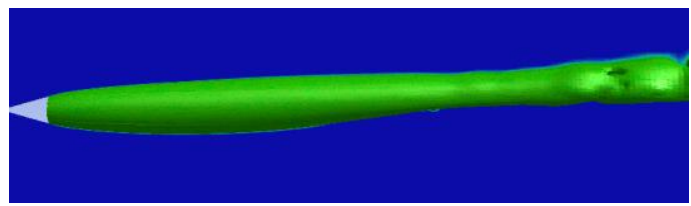

(3a) $\mathrm{S} 3 \_\bar{J}=1$

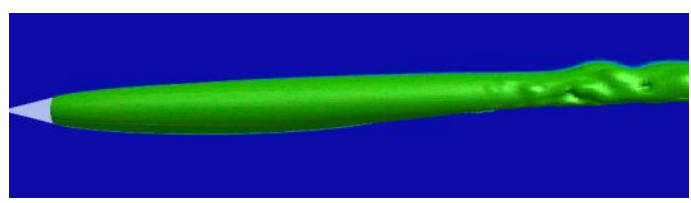

(4a) $\mathrm{S} 4 \_\bar{J}=1$

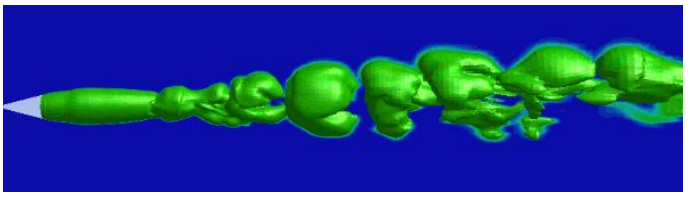

(1b) $\mathrm{S} 1 \_\bar{J}=1.88$

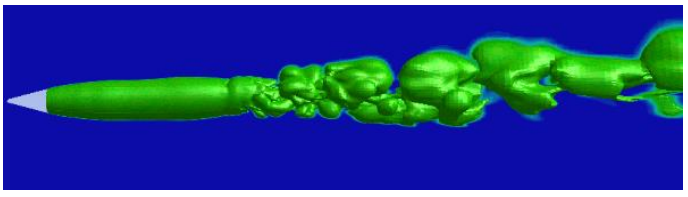

(2b) $\mathrm{S} 2 \_\bar{J}=1.88$

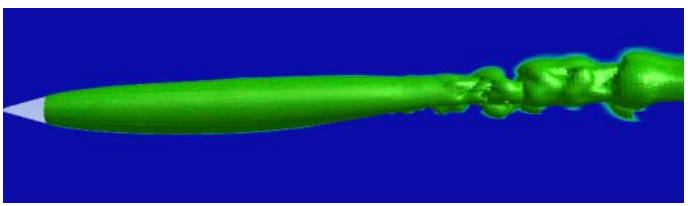

(3b) $\mathrm{S} 3 \_\bar{J}=1.88$

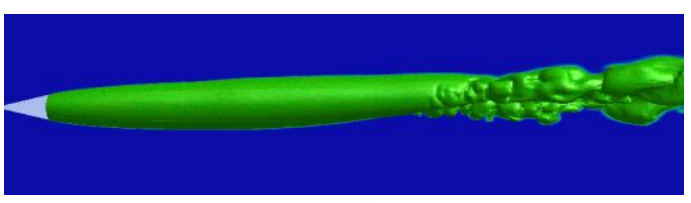

(4b) $\mathrm{S} 4 \_\bar{J}=1.88$

Fig. 5. Cavity morphology characteristics for varying relative position and jet strength at $T=0.15 \mathrm{~s}$. The cavity interface is obtained by gas volume fraction of 0.5 . Left : $\bar{P}>1$ and right : $\bar{P}<1$.

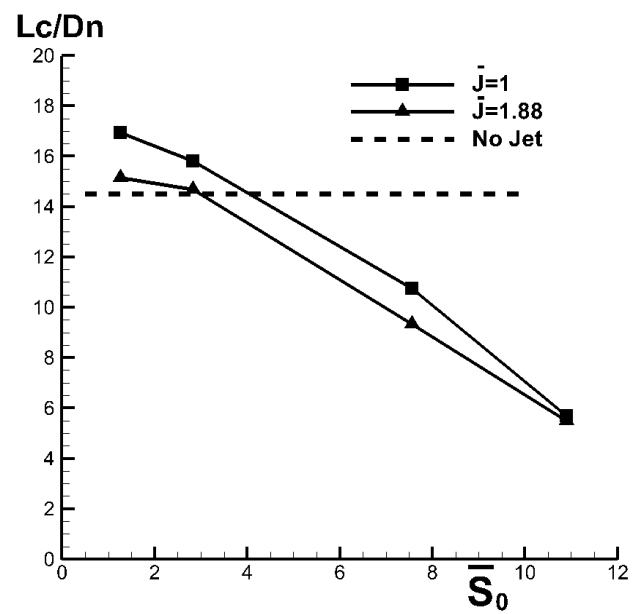

Fig. 6. Dimensionless cavity length as the distance parameter varies.

Figure 15 shows the cavity morphology characteristics for varying relative position and jet strength at $\mathrm{T}=0.15 \mathrm{~s}$. It is concluded that, when $\bar{P}>1$, the periodic gas-leakage phenomenon is not obvious, the cavity deflates in pattern of axial flow (Efros-type) so that the cavity tail looks slender; when $\bar{P}<1$, distinct periodic pulsation in the tail is observed. As for the influence of the relative position, the pinch-off phenomenon in the cavity interface evolution is observed for case $\mathrm{S} 1$ and case $\mathrm{S} 2$, but for case $\mathrm{S} 3$ and case $\mathrm{S} 4$, it turns to be not obvious. Figure 16 shows the dimensionless cavity length as the distance parameter varies. As the distance parameter $\overline{S_{0}}$ decreases, the cavity length shows a trend of increase. When the nozzle outlet is far from the cavity tail as in case S1 and case $\mathbf{S} 2$, an obvious shrinkage is resulted from the jet. However, when the nozzle moves closer to cavity tail, a slightly expansion is generated by the jet. It is 

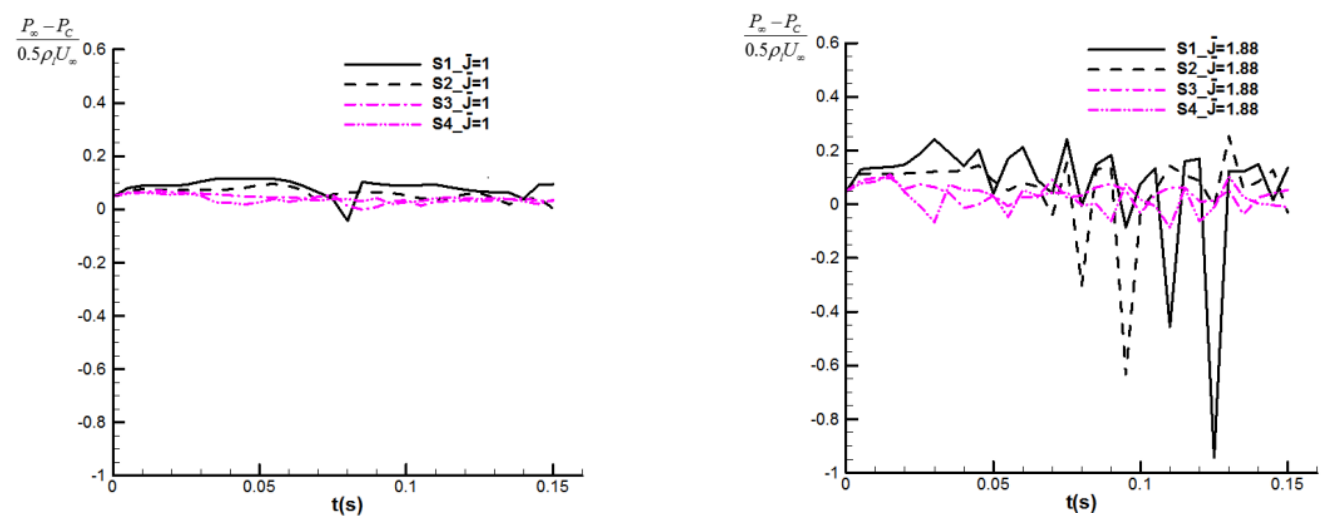

Fig. 7. Pressure pulsation characteristics inside the cavity for different relative position and jet strength.

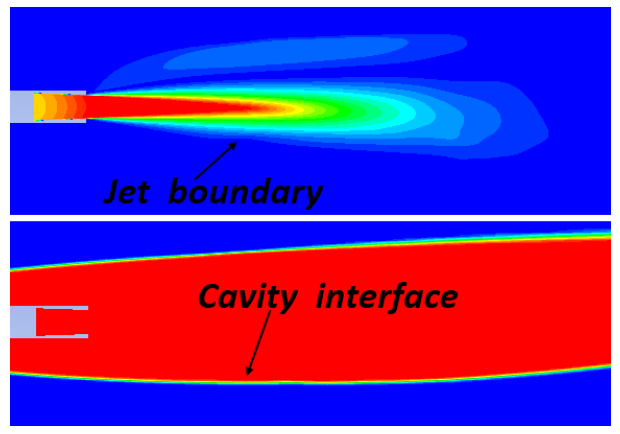

$\mathrm{S} 1 \_\bar{J}=1.88$

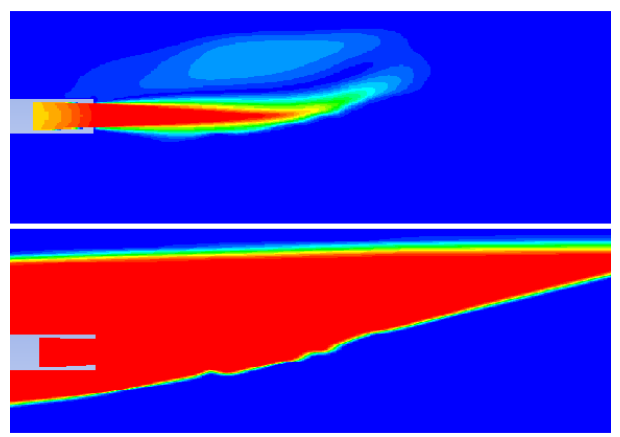

$\mathrm{S} 3 \_\bar{J}=1.88$

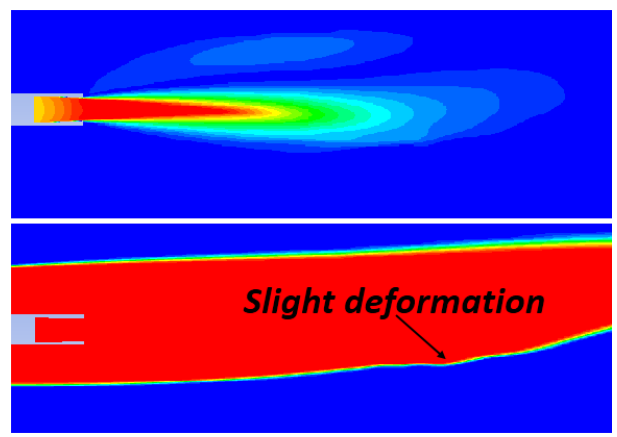

$\mathrm{S} 2 \_\bar{J}=1.88$

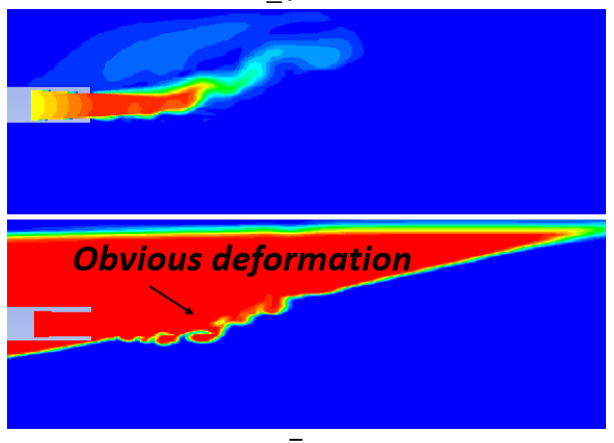

S4_ $\bar{J}=1.88$

Fig. 8. As the relative position varies, jet boundary and cavity interface interaction are showed at $\mathbf{T}=\mathbf{0 . 0 0 5}$ s.

the main reason for the variation of the cavity length whether the pinch-off occurs in the cavity interface evolution process. And the critical point $\overline{S_{0}}$ seems like to be smaller than $L_{2} / D_{n}$ in the Fig.16. From the view of the cavity instability with internal jet perturbations, case $\mathrm{S} 1$ with $\bar{J}=1.88$ is the most unstable, which has distinct interface fluctuation, and strong shrinkage. The weakest jet perturbation is in case $\mathrm{S} 4$ with $\bar{J}=1$, which has the most stable state. Figure 17 shows pressure pulsation characteristics inside the cavity for different relative position and jet strength (Pressure monitoring points are selected near the cavitator showed in Fig.9). In the process of unsteady gasleakage, the cavity would present periodic expansion and shedding. When the cavity varies in the process of expansion, the pressure would decrease. At the moment of shedding, the pressure changes quickly that behaves as a broken line for the pressure cure. When $\bar{P}>1$, the pressure fluctuation is small because the cavity is relatively stable in the axial flow gas-leakage pattern; when $\bar{P}<1$, the pressure fluctuation is really strong due to the unstable periodic pulsation pattern.

\subsection{The confined Jet Structure in a Variable Cavity}

The jet spreads inside the cavity, which will result the fluctuation and deformation of the cavity surface. On the other side, the change of the cavity will also give rise to variation of the jet style due to the change of the ambient flow parameters. Figure 18 shows the interaction between jet boundary and 


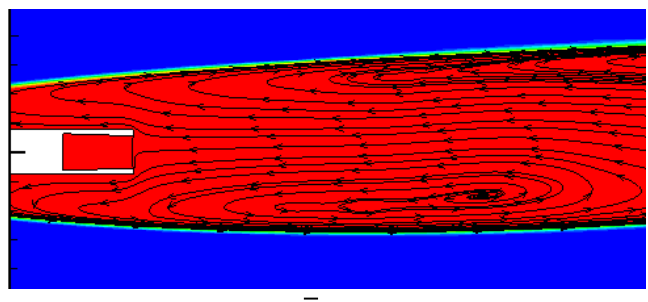

$\bar{J}=0$

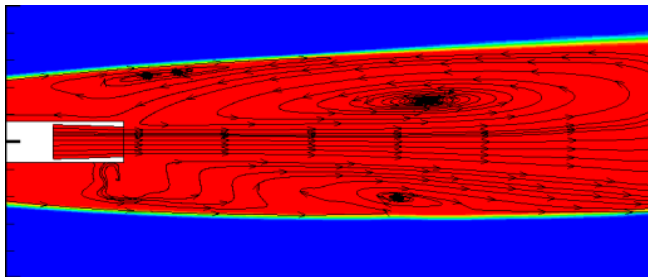

$\mathrm{S} 1 \_\bar{J}=1.88$

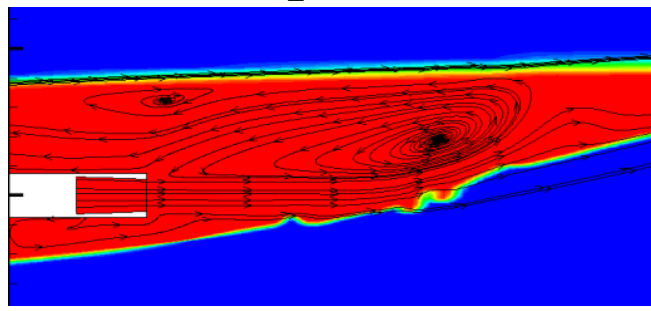

S3_ $\bar{J}=1.88$

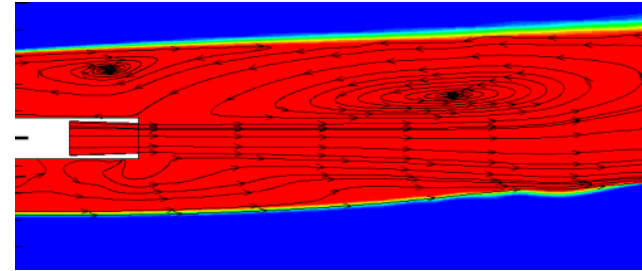

$\mathrm{S} 2{ }_{-} \bar{J}=1.88$

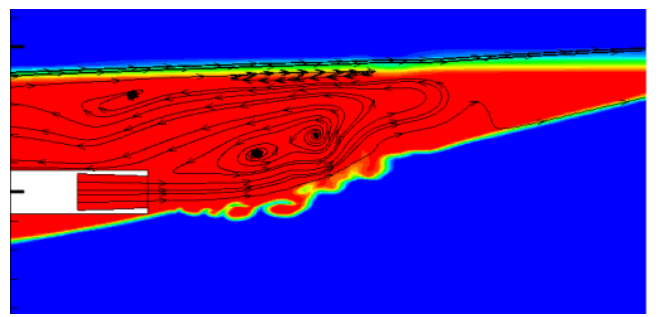

S4 $4 \bar{J}=1.88$

Fig. 9. As the relative position varies, the velocity streamline inside the cavity is showed at $T=0.005 \mathrm{~s}$.

cavity interface as the relative position varies at $\mathrm{T}=0.005 \mathrm{~s}$. As the distance parameter $\overline{S_{0}}$ decreases, jet structure turns from a symmetrical and fully developed jet into an asymmetrical and developing jet. The cavity interface evolves from no fluctuations, slight deformation, enhanced deformation to obvious deformation.

Figure 19 shows velocity streamline diagram in the cavity flow field with a gas jet as the distance parameter varies. Before the jet is generated, the gas near the gas-liquid interface is dragged by the water flow, the internal cavity appears backflow. After the jet is triggered, the internal cavity flow is dominated by the jet and the vortex direction is changed. In case $\mathrm{S} 1$, due to asymmetrical entrainment effect by the jet, the cavity has asymmetrical vortex structure, and the gas dragged by the external water flow and re-entrant jet conspire to form a small vortex in front. In case S2, the vortex in the lower side of the cavity almost disappears. In case S3 and case S4, the vortex structure is obviously limited by the narrow space. In particular, instead of a big vortex, it becomes vortex pair is in case $\mathrm{S} 4$ 。

Figure 20 shows the normalized centerline axial velocity decay of the jet for varying relative position and jet strength at $\mathrm{T}=0.005 \mathrm{~s}$. As for case $\mathrm{S} 1$ and case $\mathrm{S} 2$, the jet structure is a symmetrical and fully developed type, which is weakly disturbed from the downside cavity wall. By considering momentum balance, the entrainment effect of case $\mathrm{S} 1$ is stronger than that of case S2, so that the centreline axial mean velocity decay faster in case
$\mathrm{S} 1$. But for case S3 and case S4, the jet structure is an asymmetrical and developing type, which encounters with the stagnation effect from external water fluid, so the centreline axial mean velocity decay dramatically.

Table 3 shows the basic parameters of the confined jet structure, dimensionless potential core length and recirculation region length for varying relative position. In case $\mathrm{S} 1$, case $\mathrm{S} 2$ and case $\mathrm{S} 3$, the potential core length of the jet are almost equal to the theoretical value, but for case S4, the potential core length is smaller than the theoretical value for $0<\overline{S_{0}}<L_{1} / D_{n}$. In case $\mathrm{S} 1$ and case $\mathrm{S} 2$, the recirculation region length of the jet are approximate to the theoretical value, but for case S3 and case $\mathrm{S} 4$, the potential core length is smaller than the theoretical value for $\overline{S_{0}}<L_{2} / D_{n}$.

\subsection{Quantitative Analysis of the Entrainment Effect}

Figure 21 shows the theoretical cavity internal structure with a gas jet. $Q x$ denotes the mass flow rate across the jet section. $\dot{m}_{j e t}$ and $Q_{D}$ denotes the mass flow rate of the jet nozzle and ventilation respectively. Jet entrainment effect results in a recirculation flow in the cavity interior and builds a new gas-leakage pattern in the cavity tail. $Q_{R}$ is the reflux mass flow rate. The entrainment mass flow rate could been obtained by subtracting the mass flow at the nozzle exit from the mass flow at the jet cross section $\left(Q_{x}-\dot{m}_{\text {jet }}\right)$. 

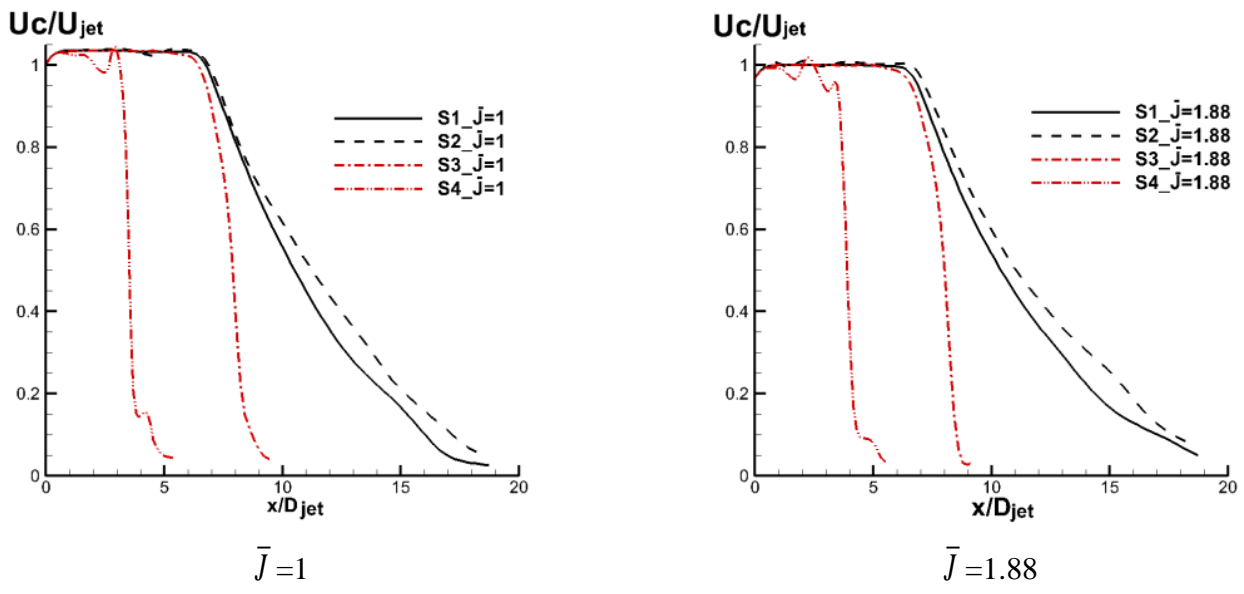

Fig. 10. Normalized centerline axial mean velocity decay at $T=0.005 \mathrm{~s}$.

Table 3 Dimensionless potential core length and recirculation region length of the jet at $T=0.005 \mathrm{~s}$

\begin{tabular}{|c|c|c|}
\hline Jet parameter & $L_{1} / D_{n}$ & $L_{2} / D_{n}$ \\
\hline Theory results & 2.19 & 6.4 \\
\hline Case 1a & 2.24 & 5.86 \\
\hline Case 1b & 2.20 & 6.20 \\
\hline Case 2a & 2.33 & 6.30 \\
\hline Case 2b & 2.30 & 6.23 \\
\hline Case 3a & 2.30 & 4.19 \\
\hline Case 3b & 2.20 & 4.31 \\
\hline Case 4a & 1.32 & 3.15 \\
\hline Case 4b & 1.29 & 3.40 \\
\hline
\end{tabular}

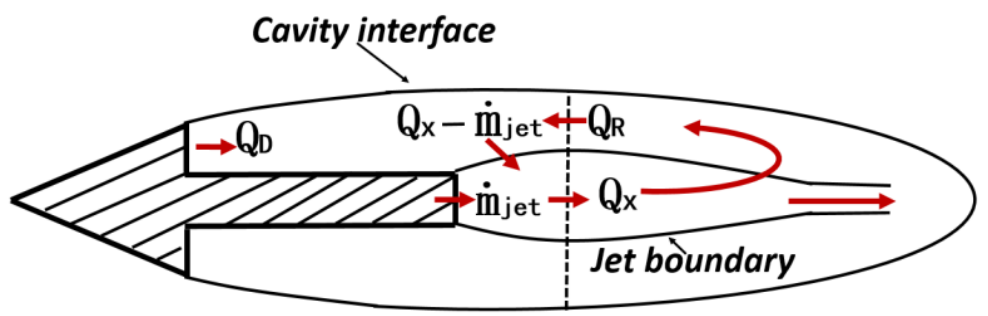

Fig. 21. Schematic of the cavity internal flow structure with a gas jet.

Figure 22 shows the normalized entrainment rates of the surrounding gas into the jet for varying relative position and jet strength at $\mathrm{T}=0.005 \mathrm{~s}$. Along the axial direction, the entrainment rate firstly increases to a peak value and then decreases. For all cases, the maximum entrainment rate appears at the maximum radius of the jet. It can be seen that the stronger the jet is, the larger the entrainment rates are. The entrainment rates also reduce with the decrease of the relative position $\overline{S_{0}}$. The nondimensional parameter $\bar{J}$ and $\overline{S_{0}}$ jointly determine the entrainment intensity.

Table 4 shows the entrainment and recirculation mass flow rate at the max jet radius for each case at $\mathrm{T}=0.005 \mathrm{~s}$. Therefore we could make a quantitative analysis to indicate the morphological changes of the cavity. Analyzing the change of gas mass in the forepart of the cavity by calculating the max entrainment flow rate plus ventilation and recirculation flow rate, we could judge whether the cavity will be depressed or expanded at the next time step. When $Q_{x}-\dot{m}_{j e t}-Q_{D}-Q_{R}>0$, it deflates the cavity so that the cavity will get smaller; when $Q_{x}-\dot{m}_{j e t}-Q_{D}-Q_{R}<0$, it inflates the cavity so that the cavity volume will become larger.

The cavity interface evolution process are also the rebuilding process from an unbalance state to a dynamic balance state, in which the beginning state is $Q_{x}-\dot{m}_{\text {jet }}-Q_{D}-Q_{R}>0$ or $<0$, and the ending 


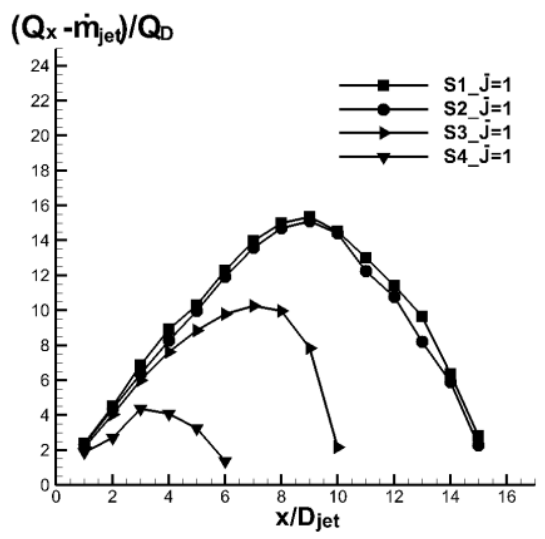

$\bar{J}=1$
$\left(Q_{x}-\dot{m}_{j e t}\right) / Q_{D}$

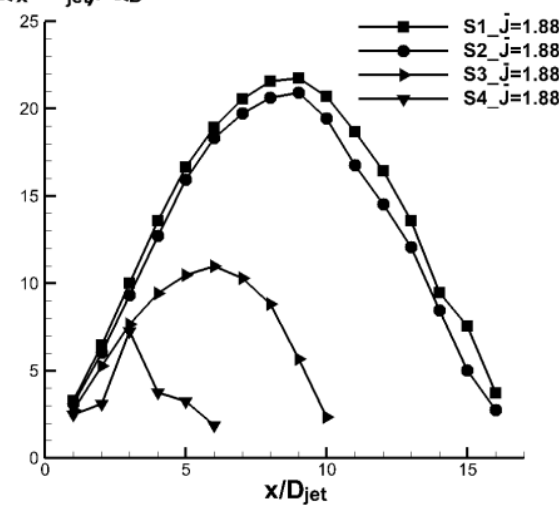

$\bar{J}=1.88$

Fig. 11. Normalized entrainment rates of the surrounding gas into the jet along the axial direction at $\mathbf{T}=\mathbf{0 . 0 0 5 s}$.

Table 4 The entrainment and recirculation mass flow rate for each case at $T=0.005 \mathrm{~s}$. The max entrainment mass flow rate is obtained at the max jet radius. The dates in the table are half of the actual value for one half of computational domain

\begin{tabular}{|c|c|c|c|c|c|}
\hline Case & $\begin{array}{c}\text { Ventilation flow } \\
\text { rate } \\
Q_{D}(\mathrm{~kg} / \mathrm{s})\end{array}$ & $\begin{array}{c}\text { Jet flow rate } \\
\dot{m}_{\text {jet }}(\mathrm{kg} / \mathrm{s})\end{array}$ & $\begin{array}{c}\text { The maximum } \\
\text { entrainment flow } \\
\text { rate } \\
Q_{x}-\dot{m}_{j e t}(\mathrm{~kg} / \mathrm{s})\end{array}$ & $\begin{array}{c}\text { Recirculation flow } \\
\text { rate } \\
Q_{R}(\mathrm{~kg} / \mathrm{s})\end{array}$ & $\begin{array}{c}Q_{x}-\dot{m}_{\text {jet }} \\
-Q_{D}-Q_{R}\end{array}$ \\
\hline $\mathrm{S} 1 \_\bar{J}=1$ & 0.00062328 & 0.00908307 & 0.00956197 & 0.0088959 & $>0$ \\
\hline $\mathrm{S} 2 \_\bar{J}=1$ & 0.00062328 & 0.00908307 & 0.00941998 & 0.0086789 & $>0$ \\
\hline $\mathrm{S} 3{ }_{-} \bar{J}=1$ & 0.00062328 & 0.00908307 & 0.00638417 & 0.0079659 & $<0$ \\
\hline $\mathrm{S} 4 \_\bar{J}=1$ & 0.00062328 & 0.00908307 & 0.00273304 & 0.0045968 & $<0$ \\
\hline $\mathrm{S} 1 \_\bar{J}=1.88$ & 0.00062328 & 0.0124656 & 0.0135627 & 0.0123124 & $>0$ \\
\hline $\mathrm{S} 2 \_\bar{J}=1.88$ & 0.00062328 & 0.0124656 & 0.0133854 & 0.0114708 & $>0$ \\
\hline $\mathrm{S} 3{ }_{-} \bar{J}=1.88$ & 0.00062328 & 0.0124656 & 0.0068512 & 0.0084827 & $<0$ \\
\hline $\mathrm{S} 4 \_\bar{J}=1.88$ & 0.00062328 & 0.0124656 & 0.0045186 & 0.0067525 & $<0$ \\
\hline
\end{tabular}

state is the mass flow balance inside the cavity that $Q_{x}-\dot{m}_{\text {jet }}-Q_{D}-Q_{R} \approx 0$ for $\bar{P}>1$, or the arithmetic mean value in one period approximately equal to zero for $\bar{P}<1$.

\section{CONCLUSION}

In order to promote the engineering application of supercavitating technology, the jet/cavity interaction was investigated based on numerical methods in this paper. A multiphase model using coupled VOF and level set method is adopted to capture gas-liquid interface. The cavity interface evolution process have been studied and summarized based on simulation and experimental dates.

A series of numerical simulations of supercavitation flows was performed to reveal the jet/cavity interaction mechanism. The transition criterion with varying jet strength and relative position is proposed and validated. Based on the numerical and theoretical analysis, it is concluded that the nondimensional parameter $\bar{J}$ and $\overline{S_{0}}$ jointly determine the entrainment effect intensity. Gas leakage ways induced by jet entrainment and cavity interface instabilities jointly determine the interface evolution mechanism. When $\bar{P}>1$, gas leaks out of the cavity in the way of the axial flow pattern; and when $\bar{P}<1$, gas leaks out of the cavity in the way of the periodic pulsation pattern. For $\overline{S_{0}}>L_{2} / D_{n}$, the cavity interface evolution experiences an expansion, necking, and shedding process; and for $\overline{S_{0}}<L_{2} / D_{n}$ the cavity wouldn't experience pinch-off.

Based on the above analysis, the supercavitation vehicles is recommended to improve the ratio of the nozzle exit area to the cavitator bottom area (increase $S_{j e t} / S_{D}$ ), make sure that the nozzle outlet is close to the cavity tail (decrease $\overline{S_{0}}$ ) and it's 
suitable for straight operation under the condition of high speed (increase $\bar{P}$ ), which tends to build a stable supercavitaion with a strong gas jet from exhaust nozzle.

Finally, a quantitative analysis method has been studied, which is to associate entrainment effect and the cavity interface evolution. In future work, we will try to construct semi-empirical formulas between cavity dimensions and $\bar{J}, \overline{S_{0}}, \mathrm{Fr}, \mathrm{Cq}$ etc, and use a regular active inflation technology to control the mass flow balance inside the cavity for the most unstable cavitation under certain circumstances $\left(\bar{P}<1\right.$ and $\left.\overline{S_{0}}>L_{2} / D_{n}\right)$. It should be an important and practical theory in the artificial supercavition technique.

\section{ACKNOWLEDGMENTS}

The authors gratefully acknowledge support by the National Nature Science Foundation of China (NSFC, Grant NO: 51776221) and the Research Project Foundation of National University of Defense Technology (Grant NO: ZK18-02-07).

\section{REFERENCES}

Albertson, M. L., Y. B. Dai, R. A. Jensen and H. Rouse (1950). Diffusion of submerged jets. Transactions ASCE. 115,639-664.

Ball, C. G., H. Fellouah and A. Pollard (2012). The flow field in turbulent round free jets. Progress in Aerospace Sciences. 501-26.

Bourlioux, A. (1995). A coupled level-set volumeof-fluid method for tracking material interfaces. In: Proceedings 6th Annual Int. Symp.on Comp. Fluid Dynamics, Lake Tahoe, USA.

Eggers, J. (1997). Nonlinear dynamics and breakup of free-surface flows. Reviews of Modern Physics 69(3),865-930.

Guzesky, L. G. (1973). Approximation dependences for axisymmetric cavities behind cones. In: Hydrodynamic Flows and Wave Processes (collected scientific papers), Inst. Thermophys, Sib. Branch, Acad. of Sci. of the USSR, Novosibirsk, 92-91.

Henderson, D., W. Pritchard and L. Smolka (1997). On the pinch-off of a pendant drop of viscous fluid. Physics of Fluids 9 (11),3188.

Kandakure, M. T., V. C. Patkar and A. W. Patwardhan (2008). Characteristics of turbulent confined jets. Chemical Engineering and Processing. 47,1234-1245.

Karn. A., R. E. A. Arndt and J. R. Hong (2016) An experimental investigation into supercavity closure mechanisms. Journal of Fluid Mechanics 789, 259-284.

Kinzel, M. P., M. H. Krane, I. N. Kirschner and M. J. Moeny (2017). A numerical assessment of the interaction of a supercavitating flow with a gas jet. Ocean Engineering 136, 304-313.

Money, M. J., M. H. Krane, I. N. Kirschner and M. P. Kinzel (2015). Jet-Supercavity Interaction: Insights from experiments. In: Proceedings of the 9th International Symposium on Cavitation (CAV2015).

Paryshev, E. V. (2006). Approximate Mathematical Models in High-Speed Hydrodynamics. Journal of Engineering Mathematics 55(1-4), 41-64.

Reichardt, H. (1946). The laws if cavitation bubbles as axially symmetrical bodies in a flow. Britian: Ministry of Aircraft Production Reports and Translations.

Schwarz, W H. (1963). The radial free jet. Chemical Engineering Science 18,779-786.

Sun, T., X. Zhang, C. Xu, G. Zhang, S. Jiang and Z. Zong (2019) Numerical modeling and simulation of the shedding mechanism and vortex structures at the development stage of ventilated partial cavitating flows. European Journal of Mechanics B-Fluids 76, 223- 232.

Sussman, M. and E. G. Puckett (2000). A coupled level set and volume-of-fluid method for computing 3D and axisymmetric incompressible two-phase flows. Journal of Computer Physics 162, 301-337.

Xu, H., C. Wang, H. Z. Lu and W. H. Huang (2018). Experimental study on submerged supersonic gaseous jet induced tail cavity. Acta Physica Sinica. 67,014703.

Zou, W., K. P. Yu and X. H. Wan (2010). Research on the gas-leakage rate of unsteady ventilated supercavity. Journal of Hydrodynamics 22(1),736-741.

Zou, W., K. P. Yu, R. E. A. Arndt, G. Zhang and Z. W. Li (2013). On the shedding of the ventilated supercavity with velocity disturbance. Ocean Engineering 57, 223-229. 